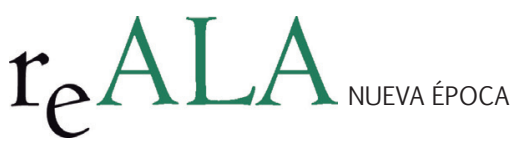

REALA, n 3, enero-junio 2015 ISSN: 1989-8975

DOI: http://dx.doi.org/10.24965/reala.voi3.10246

\title{
Propuesta de reforma del sistema electoral de Canarias
}

\author{
Adolfo López Carmona \\ Departamento de Matemática Aplicada, Escuela Técnica Superior de Ingeniería de Caminos, Canales y Puertos, Universidad de Granada \\ adolfo@ugr.es \\ Blanca Luisa Delgado Márquez \\ Departamento de Economía Internacional y de España, Facultad de Ciencias Económicas y Empresariales, Universidad de Granada
} bdelgado@ugr.es

\section{Resumen}

El actual sistema electoral canario produce discordancias entre votos recibidos y escaños asignados a los partidos debido (1) a la doble barrera electoral, (2) a que se usan las islas como circunscripciones donde se realizan repartos independientes de escaños a los partidos, (3) y a que el tamaño de estas circunscripciones no es proporcional a sus poblaciones. Ante estas discordancias proponemos como alternativa la técnica del reparto biproporcional, la cual considera tanto los votos totales que reciben los partidos así como un reparto de escaños entre circunscripciones (islas) más ajustado a la población actualizada según el último padrón de cada una de ellas.

Palabras clave

Sistema electoral, Islas Canarias, proporcionalidad, biproporcionalidad.

\section{Proposal of Reform of the electoral system of Canary Islands}

\section{Abstract}

The current canary electoral system produces discordances between the votes received and the seats allocated to parties due to (1) the double electoral barrier, (2) the use of the islands as constituencies where independent distributions of seats are made to parties, (3) and the fact that the size of these constituencies is not proportional to their populations. Given these discordances, this paper presents the technique of biproportional allocation as an alternative to the current method. Biproportionality considers both the total votes received by the parties as well as a distribution of seats among constituencies (islands) that is more adjusted to the updated population according to the last census of each one.

Key words

Electoral system, Canary Islands, proportionality, biproportionality. 


\section{1-. INTRODUCCIÓN}

Actualmente hay un creciente interés en el análisis y la reforma de sistemas electorales tanto a nivel de España en general (Pallarés Porta, 1981; Nohlen, 1983; Vallés Casadevall, 1986; Montero Gibert, 1997; Lago Peñas y Lago Peñas, 2000; Oñate Rubalcaba y Ocaña Lara, 2000; Montero Gibert y Riera Sagrera, 2008; Montero Gibert y Riera Sagrera, 2009; Gambino, 2009; Ramírez González et al., 2013; Riera Sagrera, 2013) como a nivel regional en particular (Montero Gibert y Font Fàbregas, 1991; Padró Solanet y Colomer Calsina, 1992; Lago Peñas y Montero Gibert, 2004; Delgado Ramos, 2011; Libbrecht, Maddens y Swenden, 2011; Schakel, 2011; Falcó Gimeno y Verge Mestre, 2013; Gómez Fortes y Cabeza Pérez, 2013).

En las elecciones autonómicas, Canarias se divide en siete circunscripciones (Tenerife, Gran Canaria, Lanzarote, Fuerteventura, La Palma, La Gomera, El Hierro) cada una de las cuales se corresponde con cada una de las islas (o grupo de islas) que componen el archipiélago. Canarias posee un sistema electoral con doble barrera: una a nivel regional (6\%) y otra a nivel de cada circunscripción (30\%). Cuando un partido supera al menos una de esas dos barreras o sea la lista más votada, éste forma parte del reparto de escaños. Se aplica la ley d'Hondt de forma independiente en cada circunscripción para calcular el reparto de escaños a los partidos tendiendo en cuenta dichos requisitos para participar en el reparto de escaños. De este modo, sólo serán tenidas en cuenta aquellas listas de partido o coalición que hubieran obtenido el mayor número de votos válidos de su respectiva circunscripción electoral y las siguientes que hubieran obtenido al menos el $30 \%$ de los votos válidos emitidos en la circunscripción insular o al menos el $6 \%$ de los votos válidos emitidos en la totalidad de la Comunidad Autónoma.

Así por ejemplo, con esta doble barrera, en las últimas elecciones autonómicas al Parlamento de Canarias de 2011, se dio un caso en el que varios partidos (ACSP, Verdes, UPyD,...) con más votos que el partido AHI no obtuvieron representación, mientras que AHI con menos votos obtuvo un escaño. Con lo cual se produjo discordancias entre el número total de votos y número total de escaños. Como veremos a lo largo del presente artículo, estas discordancias entre votos y escaños vienen dadas por la doble barrera, por la infrarrepresentación en número de escaños de algunas circunscripciones (Tenerife y Gran Canaria), por la sobrerrepresentación de otras (Lanzarote, Fuerteventura, La Palma, La Gomera y El Hierro) al no tenerse en cuenta su población actual para repartir los escaños a las circunscripciones, y principalmente por el hecho de hacerse los repartos independientes en cada circunscripción. Esto implica que en las cinco circunscripciones sobrerrepresentadas de tradición más nacionalista, la coalición nacionalista CC-PNC-CCN tenga ventaja sobre otros partidos de ámbito nacional (PP y PSOE) en el resultado final de escaños.

Discordancias en la representación de los partidos se han producido en todas las elecciones autonómicas de Canarias desde las primeras celebradas en 1983 hasta las últimas celebradas en 2011. No obstante este trabajo se centra en los resultados de las últimas elecciones autonómicas de Canarias de 2011 donde proponemos y aplicamos como alternativa un reparto biproporcional teniendo en cuenta votos totales para evitar tales discordancias entre votos y escaños.

Este trabajo se estructura en cinco apartados siguiendo a esta introducción. El segundo apartado recoge una breve descripción de las principales características del sistema electoral canario, las cuales provocan las actuales discordancias entre votos y escaños. El tercer apartado trata sobre el estado de la cuestión centrándonos en las modificaciones de la legislación electoral de la doble barrera electoral del sistema electoral canario desde el inicio de la democracia hasta nuestros días. En este apartado tercero también tratamos algunas de las críticas y propuestas a la doble barrera por parte de algunas formaciones políticas, plataformas de asociaciones o individuos, y particulares. En el cuarto apartado realizamos un análisis empírico de las discordancias producidas en las elecciones autonómicas de Canarias de 2011 ante las cuales proponemos como alternativa la técnica de la biproporcionalidad. En esta sección cuarta se muestra cómo las discordancias entre votos y escaños en las elecciones canarias de 2011 podrían haberse evitado si las circunscripciones electorales hubiesen tenido un tamaño proporcional a sus poblaciones, y si se hubiera aplicado la técnica de la biproporcionalidad teniendo en cuenta votos totales y no repartos independientes de escaños a los partidos. Finalmente, en el quinto y último apartado se recogen las principales conclusiones del presente estudio.

\section{2-. SISTEMA ELECTORAL CANARIO}

Las Islas Canarias son una de las diecinueve comunidades autónomas que forman España. La Comunidad Autónoma de las Islas Canarias se divide a su vez en dos provincias:

- Santa Cruz de Tenerife compuesta por cuatro islas o grupo de islas: Tenerife, la Gomera, La Palma y El Hierro.

- Las Palmas compuesta por tres islas o grupo de islas: Gran Canaria, Fuerte Ventura y Lanzarote. 
En las elecciones autonómicas al Parlamento Canario, al igual que en las elecciones al Senado, cada una de las siete islas o grupo de islas que conforman el archipiélago es una circunscripción electoral insular, tal y como se recoge en el artículo 9.4 del Estatuto de Autonomía de Canarias de 1982:

"Cada una de las islas de El Hierro, Fuerteventura, Gran Canaria, La Gomera, Lanzarote, La Palma y Tenerife constituye una circunscripción electoral."

FIGURA 1: MAPA DE LAS ISLAS CANARIAS

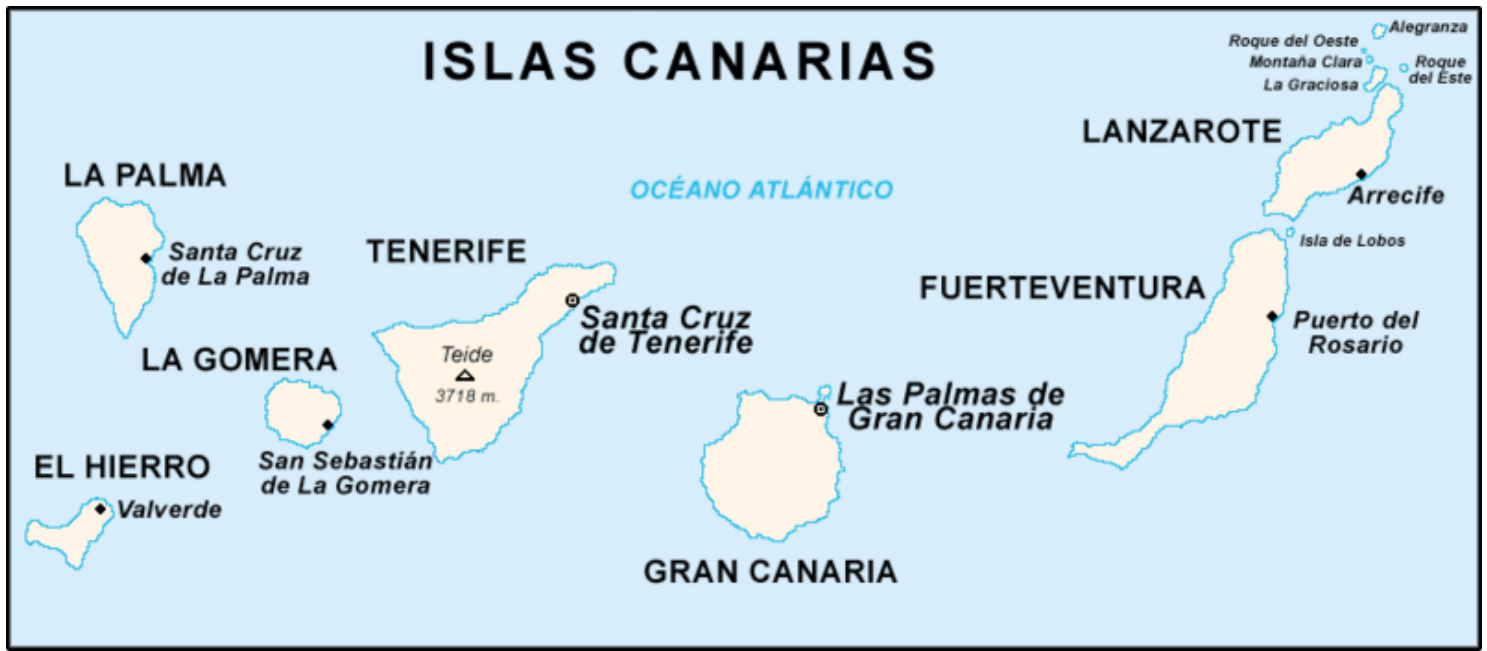

Fuente: Wikimedia Commons.

Sin embargo, para las elecciones al Congreso, las dos provincias canarias coinciden con las dos circunscripciones electorales.

El actual sistema electoral canario se rige por la Ley de Elecciones al Parlamento de Canarias de 2003 que a su vez se basa en el Estatuto de Autonomía de Canarias de 1982. Según el artículo 9.2 del Estatuto de Autonomía “el sistema electoral es el de representación proporcional”. El Parlamento Canario está compuesto en la actualidad por 60 diputados, número que según el artículo 9.3 de dicho Estatuto de Autonomía no será inferior a 50 ni superior a 70. De estos 60 diputados, se distribuyen 30 para cada una de las dos provincias. Y los 30 diputados que corresponden a cada provincia a su vez se distribuyen entre sus circunscripciones según la siguiente Tabla 1:

TABLA 1: DISTRIBUCIÓN ACTUAL DE ESCAÑOS ENTRE LAS 7 CIRCUNSCRIPCIONES

\begin{tabular}{|c|l|r|r|}
\hline \multicolumn{1}{|c|}{ Provincia } & \multicolumn{1}{|c|}{ Circunscripción } & Población & Escaños \\
\hline \multirow{5}{*}{ Santa Cruz de Tenerife } & El Hierro & 10960 & 3 \\
\cline { 2 - 4 } & La Gomera & 22776 & 4 \\
\cline { 2 - 4 } & La Palma & 87324 & 8 \\
\cline { 2 - 4 } & Tenerife & 906854 & 15 \\
\cline { 2 - 4 } & Total & 1027914 & 30 \\
\hline \multirow{5}{*}{ Las Palmas } & Fuerteventura & 103492 & 7 \\
\cline { 2 - 4 } & Lanzarote & 141437 & 15 \\
\cline { 2 - 4 } & Gran Canaria & 845676 & 30 \\
\cline { 2 - 4 } & Total & 1090605 & 60 \\
\cline { 2 - 4 } & TOTAL & 2118519 & 3 \\
\hline
\end{tabular}

Fuente: Elaboración propia a partir de Padrón de 2010 (www.ine.es) y página oficial del Parlamento de Canarias (www.parcan.es)

Este reparto de escaños entre las circunscripciones está descrito en la disposición transitoria 1.1 del Estatuto de Autonomía de Canarias de la siguiente manera:

“De acuerdo con lo establecido en el artículo 9 del presente Estatuto, y en tanto no se disponga otra cosa por una Ley del Parlamento Canario aprobada por mayoría de dos terceras partes de sus 
miembros, se fija en sesenta el número de diputados del Parlamento Canario, conforme a la siguiente distribución: quince por cada una de las islas de Gran Canaria y Tenerife, ocho por La Palma, ocho por Lanzarote, siete por Fuerteventura, cuatro por La Gomera y tres por El Hierro."

En la disposición transitoria 1.2 del Estatuto de Autonomía se establece una doble barrera electoral tanto a nivel regional así como una barrera a nivel de circunscripción para formar parte del reparto de escaños:

"De acuerdo con lo establecido en el artículo 9 del presente Estatuto, y en tanto no se disponga otra cosa por una Ley del Parlamento Canario aprobada por mayoría de dos terceras partes de sus miembros, se establece que sólo serán tenidas en cuenta aquellas listas de partido o coalición que hubieran obtenido el mayor número de votos válidos de su respectiva circunscripción electoral y las siguientes que hubieran obtenido, al menos, el 30 por 100 de los votos válidos emitidos en la circunscripción insular o, sumando los de todas las circunscripciones en donde hubiera presentado candidatura, al menos, el 6 por 100 de los votos válidos emitidos en la totalidad de la Comunidad Autónoma."

En esta disposición queda claro que un partido forma parte del reparto si en una circunscripción concreta es el más votado, o al menos alcanza el $30 \%$ de los votos a nivel insular o el $6 \%$ a nivel regional. Esta doble barrera, el reparto de escaños a las circunscripciones sin tener en cuenta sus poblaciones actualizadas, y sobre todo hacer repartos independientes de escaños a los partidos en cada circunscripción son las principales causas de las actuales discordancias entre votos y escaños. Así tenemos casos de circunscripciones donde partidos con muchos más votos que otros no forman parte del reparto de escaños por no alcanzar el 30\% de los votos en esa circunscripción ni a nivel regional alcanzar la barrera del 6\%. Tal es el caso, por ejemplo, de la circunscripción de Lanzarote donde en las elecciones de 2007 la coalición Partido de Independientes de Lanzarote/Centro Canario Nacionalista (PIL(CN) obtuvo más votos que Coalición Canaria/Partido Nacionalista Canario y sin embargo no formó parte del reparto de escaños en esa circunscripción, mientras que Coalición Canaria/Partido Nacionalista Canario (CC-PNC) obtuvo dos escaños (Tabla 2). Es decir, la coalición PIL-CCN no formó parte del reparto por no superar ni la barrera a nivel de circunscripción del 30\% (Tabla 2) ni la barrera global del 6\% (Tabla 3).

TABLA 2. VOTOS Y ESCAÑOS ACTUALES EN LA CIRCUNSCRIPCIÓN DE LANZAROTE. ELECCIONES 2007

\begin{tabular}{|l|r|r|r|}
\hline \multicolumn{1}{|c|}{ Partido } & \multicolumn{2}{c|}{ Votos } & \multicolumn{2}{c|}{ \% Votos } & Escaños \\
\hline PSOE & 12727 & 29,48 & 4 \\
\hline PIL-CCN & 9701 & 22,47 & 2 \\
\hline CC-PNC & 8303 & 19,23 & \\
\hline PP & 6751 & 15,64 & \\
\hline NC-PNL & 2863 & 6,63 & \\
\hline AC25M-APCa & 1252 & 2,90 & 7 \\
\hline ISAL & 870 & 2,02 & \\
\hline Verdes & 420 & 0,97 & \\
\hline IUC & 284 & 0,66 & 100 \\
\hline Total & 43171 & & \\
\hline
\end{tabular}

Fuente: Elaboración propia a partir de página oficial del Parlamento de Canarias (www.parcan.es)

TABLA 3. ELECCIONES 2007 AL PARLAMENTO DE CANARIAS

\begin{tabular}{|c|c|c|c|}
\hline Partido & Votos & $\%$ Votos & Escaños \\
\hline PSOE & 322833 & 35,01 & 26 \\
\hline PP & 224883 & 24,39 & 15 \\
\hline CC-PNC & 222905 & 24,17 & 17 \\
\hline NC-NGC & 46303 & 5,02 & \\
\hline $\mathrm{CCN}$ & 36975 & 4,01 & \\
\hline Verdes & 17793 & 1,93 & \\
\hline PIL-CCN & 9701 & 1,05 & \\
\hline $\mathrm{CGCa}$ & 8512 & 0,92 & \\
\hline IUC & 6558 & 0,71 & \\
\hline
\end{tabular}




\begin{tabular}{|l|r|r|r|}
\hline APCa-AC25M & 3572 & 0,39 & 2 \\
\hline CC-AHI & 2973 & 0,32 & \\
\hline Otros & 19215 & 2,08 & 60 \\
\hline Total & 922223 & 100 & \\
\hline
\end{tabular}

Fuente: Elaboración Propia a partir de Página Oficial del Parlamento de Canarias (www.parcan.es)

\section{3-. ESTADO DE LA CUESTIÓN}

\section{1-. Antecedentes}

La Ley Orgánica 10/1982 establecía una doble barrera electoral en el Artículo 8.2:

"El sistema electoral es el de representación proporcional. No serán tenidas en cuenta aquellas listas de partido o coalición que no obtengan, al menos, el tres por ciento de los votos válidos emitidos en la Región o el veinte por ciento de los válidamente emitidos en la circunscripción electoral.”

Esta doble barrera establecida por la Ley Orgánica 10/1982 fue modificada por la Ley Orgánica 4/1996, la cual aumentó la barrera regional del 20\% al 30\% y la barrera en cada circunscripción del 3\% al 6\%.

La reforma del estatuto de autonomía canario introducida por la Ley Orgánica 4/1996 llevó a cabo importantes modificaciones en el sistema electoral de Canarias, cuya constitucionalidad quedó confirmada y ratificada por la Sentencia del Tribunal Constitucional 225/1998 de 26 de noviembre, publicada en BOE el 30 de diciembre. Este tipo de subsistema electoral resultó polémico desde su aprobación. De hecho, la tramitación de la reforma del estatuto canario fue aprobada por unanimidad en casi todos sus puntos, tanto en el Parlamento Canario como en las Cortes Generales, excepto en lo referente al sistema electoral, que sólo tenía el apoyo del Partido Popular y Coalición Canaria (CC) que por aquella época eran socios de Gobierno a nivel regional y nacional. Por el contrario, se oponían las demás fuerzas políticas del parlamento autonómico canario: Partido Socialista (PSC-PSOE), Plataforma Canaria Nacionalista (PCN) y Agrupación Herrera Independiente ( $\mathrm{AHI}$ ). En el Congreso de los Diputados, se oponían a dicha propuesta Izquierda Unida (IU), PSOE y Agrupación Herrera Independiente (AHI) (Pérez Alberdi, 2001).

En el año 1997 el Defensor del Pueblo interpuso un recurso de inconstitucionalidad (1.324/1997) contra el párrafo segundo de la Disposición transitoria primera de la Ley Orgánica 4/1996, de 30 de diciembre, de reforma de la Ley Orgánica 10/1982, de 10 de agosto, del Estatuto de Autonomía de Canarias. Aunque dicho recurso se basa sobre varias líneas de razonamiento, vamos a citar a continuación algunas de las más importantes. Una de las principales líneas de razonamiento, entre otras, hace referencia a la infracción de la representación proporcional por los criterios de la lista más votada y de la doble barrera electoral:

“El precepto impugnado infringe el principio de representación proporcional establecido en los arts. 152.1 C.E. y 9 del Estatuto de Autonomía de Canarias. Por una parte, la inclusión del criterio de la lista más votada en la circunscripción supone introducir un elemento característico del sistema mayoritario en detrimento del principio de representación proporcional exigido por el art. 152.1 C.E. y por el art. 9 del Estatuto de Autonomía de Canarias.

De otra parte, las nuevas barreras o topes electorales que el precepto recurrido establece a los efectos de la asignación de escaños, elevándolos del 3 al 6 por 100, respecto del ámbito electoral de toda la Comunidad Autónoma, y del 20 al 30 por 100 por referencia a los votos válidos emitidos en cada circunscripción insular, implican, de hecho, la eliminación de cualquier dimensión proporcional en el sistema electoral que, por esta vía, se convierte en decididamente mayoritario."

De la anterior línea de razonamiento se deriva la siguiente también sobre la barrera electoral, la cual citamos literalmente a continuación:

“Conculcación del valor «pluralismo político» reconocido en el art. 1.1. C.E. Sostiene el Defensor del Pueblo que las barreras electorales establecidas en la norma recurrida reducirán notablemente el número de partidos con representación parlamentaria. En efecto, dichas barreras no parecen perseguir el fin constitucionalmente lícito de evitar una excesiva fragmentación política. Antes bien, son un medio de reforzar y amplificar artificialmente las mayorías políticas actualmente existentes a costa de hacer desaparecer del arco parlamentario a las minorías en él representadas." 
Finalmente la Sentencia Tribunal Constitucional desestimó dicho recurso de inconstitucionalidad y justifica de manera explícita la actual doble barrera electoral. Por un lado justifica la barrera regional del $6 \%$ con el siguiente argumento:

"Esa misma circunstancia, sólo explicable desde las peculiaridades propias de una Comunidad Autónoma insular como la de Canarias, es la que sitúa la indicada barrera del 6 por 100 en el límite de lo constitucionalmente tolerable, pues, ese incremento porcentual y la correlativa reducción de posibilidades de acceso al escaño para las fuerzas políticas minoritarias se corrige, en cierto modo, en las islas menores, por el trato favorecedor de que son objeto las minorías político-territoriales mediante las otras cláusulas contenidas en el mismo precepto legal. En cuanto a las islas mayores, es cierto que la barrera electoral excede en un punto a la barrera del 5 por 100 de los votos válidos en la Comunidad Autónoma, cuya legitimidad este Tribunal ya ha reconocido; pero esta diferencia no es suficiente para que este Tribunal, que no ha elevado a categoría un determinado porcentaje numérico, declare la inconstitucionalidad de tal barrera electoral apreciada en el conjunto del sistema electoral canario. Por todo lo expuesto, no cabe estimar la invocada vulneración del derecho a la igualdad ni de la vertiente fundamental del derecho de sufragio pasivo."

Por otra parte, la Sentencia Tribunal Constitucional también justifica la barrera insular del 30\% de la siguiente manera:

"Barreras o cláusulas de exclusión que, en el caso de la Comunidad Autónoma de Canarias, no tienen por exclusivo objeto restringir los efectos proporcionales de la fórmula electoral utilizada, en beneficio de otros criterios favorables a la gobernabilidad de la Comunidad Autónoma, como pueden ser evitar la excesiva fragmentación política de la Cámara autonómica, o favorecer la estabilidad gubernamental. En rigor, a esa finalidad, únicamente responde la denominada «barrera regional» ahora situada en el 6 por 100 de los votos válidos emitidos. Contrariamente, el fin perseguido por la «barrera local o de la circunscripción»-que la norma impugnada sitúa en el 30 por 100-es el de asegurar la presencia de fuerzas políticas mayoritarias en la circunscripción, pero minoritarias en el conjunto de la Comunidad Autónoma. Su objetivo no es, pues, el de excluir a la minorías, sino el de asegurar su presencia por razón del territorio. De este modo, si la primera de las mencionadas cláusulas de exclusión («barrera regional») favorece a las fuerzas políticas mayoritarias en la Comunidad Autónoma en favor de la gobernabilidad y estabilidad, la segunda supone un correctivo de la primera en beneficio de la representación de cada isla, permitiendo la presencia en el Parlamento canario de candidaturas de fuerte implantación insular, aunque con escaso nivel de voto en su valoración global desde la óptica del voto emitido en el conjunto de la Comunidad Autónoma."

\section{2-. Propuestas de reforma del sistema electoral canario}

Hasta el momento hay poca literatura que critique o/y proponga alternativas al actual sistema electoral canario. No obstante, se pueden encontrar algunas breves notas de prensa en Internet de partidos minoritarios canarios o de corrientes de opinión que critican el actual sistema electoral canario proponiendo alternativas genéricas sin métodos concretos.

\subsection{1-. Proposición de Ley para la Democratización del Sistema Electoral al Parlamento de Canarias}

Esta Proposición Ley de 22 de diciembre de 2010 (Boletín Oficial del Parlamento de Canarias) comienza criticando de manera explicita la doble barrera electoral:

“La regulación del sistema de elección de los diputados al Parlamento de Canarias es la que resulta del propio Estatuto de Autonomía, cuyos artículos 9 y disposición transitoria primera, apartados $1^{\circ}$ y $2^{\circ}$, definen el número de diputados y su distribución, la circunscripción electoral, de carácter insular, y la exigencia de que las candidaturas obtengan al menos un treinta por ciento de los votos válidos en la circunscripción insular, o resultar la candidatura más votada, o el seis por ciento de los votos válidos emitidos en la totalidad de la Comunidad Autónoma, sumando los de todas las circunscripciones insulares donde se hubiera presentado el partido o coalición, para ser tomados en cuenta a la hora de la distribución de escaños.

El carácter tan restrictivo de dichas barreras electorales ha supuesto una clara limitación del pluralismo político y territorial, al dificultar el acceso al Parlamento de fuerzas políticas que pueden 
llegar a representar a casi la tercera parte de los votantes de una isla."

Como hemos visto en el apartado dos, la doble barrera puede provocar discordancias dentro de cada circunscripción consistentes en que en esa circunscripción un partido con más votos que otro no forma parte del reparto de escaños, y partidos con menos votos sí forman parte del reparto e incluso pueden llegar a conseguir escaño. La propuesta de ley ejemplifica de la siguiente manera dicho desequilibrio poniendo dos ejemplos concretos (Tablas 4 y 5 ):

"Y desnaturaliza la propia circunscripción insular, que es la base del mismo sistema electoral, al posibilitar que pueda obtener escaño por una isla aquella candidatura de partido o coalición que, aun siendo menos votada en dicha isla que otra candidatura que por aplicación de la barrera electoral no haya obtenido representación parlamentaria, haya logrado más del seis por ciento en la totalidad de la Comunidad Autónoma. Esta posibilidad se ha materializado ya en la realidad, en las circunscripciones de Lanzarote (1999) y Gran Canaria (2007), alterando de una forma injustificada las preferencias de los electores de la isla al efectuar el reparto de los escaños."

TABLA 4. CIRCUNSCRIPCIÓN DE LANZAROTE. ELECCIONES 1999

\begin{tabular}{|l|r|r|r|}
\hline \multicolumn{1}{|c|}{ Partido } & Votos & \% Votos & Escaños \\
\hline CC & 10424 & 28,49 & 4 \\
\hline FNC- PIL & 10314 & 28,19 & 3 \\
\hline PSOE & 8855 & 24,21 & 1 \\
\hline PP & 4858 & 13,28 & 3,05 \\
\hline IUC & 1116 & 0,40 & \\
\hline UC-CDS & 147 & 2,37 & \\
\hline Votos en blanco & 868 & 100 & \\
\hline Total & 36582 & & \\
\hline
\end{tabular}

Elaboración Propia: Elaboración propia a partir de Proposición Ley de 22 de diciembre de 2010

TABLA 5. CIRCUNSCRIPCIÓN DE GRAN CANARIA. ELECCIONES 2007

\begin{tabular}{|l|r|r|r|}
\hline \multicolumn{1}{|c|}{ Partido } & Votos & \% Votos & Escaños \\
\hline PSOE & 149183 & 37,85 & 7 \\
\hline PP & 134744 & 34,19 & 11,75 \\
\hline NC & 46303 & 5,41 & 1 \\
\hline CC-PNC & 21338 & 9,46 & 1,33 \\
\hline Otros & 37286 & 100 & 15 \\
\hline Votos en blanco & 394095 & 241 & 1 \\
\hline Total & 394010 \\
\hline
\end{tabular}

Elaboración Propia: Elaboración propia a partir de Proposición Ley de 22 de diciembre de 2010

Por último, esta Propuesta de Ley también hace una breve mención al que se podría considerar como una de las grandes debilidades del sistema electoral canario, el reparto no proporcional de escaños entre las circunscripciones:

"Hay otros aspectos del sistema electoral, como el del número de diputados, su distribución más ajustada entre las islas en base al criterio poblacional o la creación, junto a las circunscripciones insulares, de una circunscripción de ámbito general o autonómico, de indudable interés, que deberán ser planteados legislativamente cuando reúnan los necesarios apoyos políticos y territoriales."

\subsection{2.- Propuesta de reforma del Estatuto de Autonomía de Canarias de 2006}

El 22 de septiembre de 2006 se publicó en el BOE una proposición de ley presentada por el Parlamento de Canarias Ilamada "Propuesta de reforma del Estatuto de Autonomía de Canarias". La disposición transitoria única de dicha propuesta de reforma, plantea reducir ambas barreras (insular y regional) a la mitad: 
"A efectos de la elección en las circunscripciones insulares, sólo serán tenidas en cuenta aquellas

listas de partido o coalición que hubieran obtenido, al menos, el 15\% de los votos válidos de su respectiva circunscripción insular, o, sumando los de todas las circunscripciones insulares hubieran obtenido, al menos, el 3\% de los votos válidos emitidos en la totalidad de la Comunidad Autónoma."

Por el momento, esta propuesta de reforma del estatuto de autonomía de Canarias, está aún pendiente de aprobación por el Congreso de los Diputados.

\subsection{3-. Propuestas de formaciones políticas, plataformas de ciudadanos y particulares}

\section{Partido Socialista Obrero Español (PSOE)}

Este partido propone la creación de una circunscripción regional única. Otros dos aspectos que consideran que deberían ser modificados necesariamente son (PSOE, 2009):

- La distribución de escaños entre las islas, para acercarse al principio de que el voto de cada ciudadano valga lo mismo en todas las circunscripciones (islas). Esto requiere que las islas más pobladas con el $80 \%$ de la población total les corresponda un mayor número de escaños. Resolver esta cuestión no es fácil porque las islas no capitalinas tienen más escaños de los que les corresponderían según su población con la intención de favorecerlas de manera intencionada. Para cambiar esta situación haría falta un consenso territorial y entre los partidos políticos.

- Rebajar las barreras electorales aplicando una fórmula proporcional tal y como se refleja en la Constitución y en el Estatuto de Autonomía. Proponen eliminar por completo la barrera regional del 6\% y rebajar la barrera insular del $30 \%$ al $5 \%$.

\section{Centro Canario Nacionalista (CCN)}

Esta formación política critica que las barreras electorales en Canarias son las más altas de Europa y sirven para mantener la hegemonía de los tres partidos mayoritarios y para que 150 mil canarios no vean reflejados sus intereses políticos en el Parlamento de Canarias.

Ante esto, CCN defiende como única barrera el 5\% en cada circunscripción (isla) y ninguna barrera a nivel regional. Para el reparto de escaños a los partidos se usaría el método d'Hondt por ser un método proporcional de reparto de escaños según los votos obtenidos.

También proponen la reforma del Estatuto de Autonomía para reducir a la mitad los 60 diputados autonómicos actuales y para elaborar una nueva ley electoral.

En palabras del propio Benito Codina, portavoz parlamentario de CCN, "la representatividad es un requisito de las instituciones democráticas y el Parlamento Canario, desgraciadamente, no la tiene en estos momentos, por el injusto y desproporcionado sistema electoral que deja fuera a una gran parte de los intereses políticos de los canarios. Esto lo dice un partido con representación parlamentaria" (Centro Canario Nacionalista, 2013).

\section{Nueva Canarias (NC)}

NC tiene en cuenta los desequilibrios en el reparto de escaños a las circunscripciones donde reconocen que salen perjudicadas las islas más pobladas. No obstante, no quieren abordar esta cuestión y prefieren centrarse tan sólo en proponer una modificación de la doble barrera: eliminar la barrera regional del 6\% y dejar como única barrera la insular que bajaría de $30 \%$ al $5 \%$ de los votos, tal y como también propone CCN en el apartado anterior (Canarias Ahora, 2013).

\section{Izquierda Unida Canaria (IUC)}

Ramón Trujillo (Canarias Ahora, 2013), coordinador de IUC, afirmó que "el Parlamento de Canarias tendrá la posibilidad de democratizarse más si abre el camino a la propuesta de rebajar las barreras electorales al $5 \%$, tal y como propone Nueva Canarias en su propuesta, que es insuficiente, pero supondría un cierto avance. Sin embargo, la propuesta no prosperará porque PP, CC y PSOE siempre condicionan las reformas electorales a cuestiones que saben que no saldrán adelante en la legislatura".

\section{Mario Marcelo Regidor Arenales}

La propuesta de reforma del sistema electoral canario de Mario Marcelo Regidor Arenales (Nueva Tribuna, 2013) se basa en tres aspectos que citamos a continuación literalmente: 
“...

El número de diputados debería elevarse a 70. La explicación es muy sencilla: todo manual de ciencia política que estudie la representatividad de la sociedad en las instituciones por medio de elecciones, coincide en que, a mayor número de legisladores, mayor proporcionalidad existirá y mayores serán las probabilidades de que cada una de las sensibilidades presentes en dicha sociedad encuentre cabida en el Parlamento.

La barrera electoral insular, que se sitúa en el 30\% del cuerpo electoral de la isla de referencia, debería desaparecer. Únicamente favorece los insularismos y no somos un conjunto de islas, sino una región con las peculiaridades propias de cada una, pero con un destino común y una visión de progreso, que debe plasmarse en un legislativo que tenga a Canarias como referente principal. No obstante, en previsión de que una excesiva fragmentación del hemiciclo haga ingobernable nuestra comunidad, creo que debe mantenerse una barrera mínima a nivel regional que podría ser del $5 \%$, mejorando la barrera actual del 6\% consagrada en el Estatuto.

Por último, y quizá lo que más controversia podría suscitar, debe abandonarse la circunscripción insular y apostar por una única circunscripción de carácter regional. Es decir, no se elegirían a los diputados por isla siguiendo el esquema de la triple paridad sino mediante una única lista regional presentada por cada partido. Es cierto que todas las islas deberían estar representadas en el Parlamento en razón de su población pero en ningún lado se dice que tenga que ser por circunscripciones insulares.

$$
\ldots "
$$

\section{Demócratas para el Cambio}

Demócratas para el Cambio no es un partido político sino una plataforma de organizaciones e individuos que buscan cambiar el sistema electoral en dos aspectos fundamentales aunque sin proponer medidas o métodos concretos alternativos (Demócratas para el Cambio, 2007):

- Mejorar la proporcionalidad, incrementando la representación de las circunscripciones capitalinas, pero no especifican qué método de reparto de escaños a las circunscripciones propondrían.

- Reducir las barreras electorales a una con el tope máximo del 5\%, pero no detallan cuál de las dos barreras dejarían, si la regional o la insular. Lo único que especifican es que la única barrera que quedara (regional o insular) fuese como máximo del $5 \%$.

\section{4-. BIPROPORCIONALIDAD CON BARRERA ELECTORAL: PROPUESTA DE REFORMA DEL SISTEMA ELECTORAL CANARIO}

\section{1-. Barrera electoral}

El uso de barreras electorales tiene sentido en aquellos sistemas electorales donde los tamaños de las circunscripciones electorales son grandes. En estos casos si no se usara una barrera electoral habría un gran riesgo de fragmentación del parlamento con la consecuente ingobernabilidad.

Las diversas propuestas tanto de investigadores como de partidos políticos apuestan por barreras del 3\% de los votos que han sido muy usadas a lo largo del tiempo. Pero pensamos que no son razonables ni necesarias en muchas ocasiones ya que en los casos de circunscripciones pequeñas el método d'Hondt conlleva barreras implícitas mayores que el 3\%. Sin embargo, en el caso de Canarias una barrera del 3\% a nivel regional sí surtiría efecto y sería recomendable si el conjunto de Canarias actuara como una única circunscripción. El 3\% de 60 sería 1,8 escaños que con el método de Sainte-Laguë supondrían dos escaños y con el método d'Hondt supondrían uno o dos escaños, los cuales irían a parar a los partidos que superen dicha barrera del 3\% (Tabla 7). Incluso estos dos escaños podrían representar una prima importante para la gobernabilidad, yendo a parar a los partidos más votados. Además se consigue un reparto más equitativo y se evitan discordancias en las que partidos con muchos más votos que otros no reciben ningún escaño. Basta comparar en la Tabla 7 la columna tercera, donde aparecen las cuotas, con la columna última donde aparece el reparto propuesto. Con nuestro método es evidente que al asignar los escaños a los partidos según sus votos totales jamás ocurre una discordancia entre votos totales y escaños totales recibidos por los partidos, es decir, no es posible que un partido con menos votos que otro reciba más escaños. 
Sila barrera usada hubiese sido más pequeña, la prima al partido vencedor habría disminuido significativamente, lo cual perjudicaría la gobernabilidad. En tales casos podría ser recomendable realizar la asignación de los escaños a los partidos en dos etapas, de forma que la última etapa sea muy beneficiosa para el vencedor (Ramírez González y Márquez García, 2010).

\section{2-. Reparto biproporcional}

Una manera de evitar que se produzcan discordancias es que siempre los escaños totales de cada partido se obtengan en función de sus votos totales. Aunque al final es necesario distribuir los escaños totales de cada partido entre las circunscripciones, y para ello la técnica de la biproporcionalidad (Balinski y Demange, 1989) es uno de los métodos más adecuados y ya aplicados anteriormente (Ramírez González, Pukelsheim, Palomares Bautista y Martínez Aroza, 2008; Ramírez González, 2010; Ramírez González y Márquez García, 2010; Ramírez González et al., 2013).

Comenzamos hallando el tamaño de las circunscripciones de manera que damos dos escaños iniciales a cada isla por el mero hecho de ser una circunscripción, ya que si no diéramos estos dos escaños iniciales la isla de El Hierro quedaría sin escaño, puesto que su cuota de población respecto del total de la población canaria sería del 0,31, y por tanto, al redondear con el método de Sainte-Laguë quedaría sin representación. En nuestra propuesta vamos a mantener los 30 escaños actuales para cada una de las dos provincias, los cuales se repartirán a su vez a cada isla (Tabla 6).

El reparto de escaños entre las circunscripciones se haría usando el método de Webster (también llamado de Sainte-Laguë), que redondea las fracciones al entero más próximo, lo notamos por $[\cdot]_{w}$. Así, $[3.62]_{w}=4,[3.49]_{w}=3$.

La cuota o proporción exacta de escaños (Tabla 6) que corresponde a cada una de las siete circunscripciones (islas) se obtiene de la siguiente manera:

$$
Q_{i}=\boldsymbol{\theta} \times \frac{P_{i}}{\mathbb{P}}
$$

Siendo:

$Q_{i}=$ cuota de escaños que correspondería proporcionalmente a la isla i por su población

60 = escaños a repartir proporcionalmente entre las siete circunscripciones

$P_{i}=$ población de la isla $i$

PT = población total de las Islas Canarias

Para calcular los escaños que corresponden a cada una de las cuatro circunscripciones de la provincia de Santa Cruz de Tenerife mediante el método Webster y teniendo en cuenta el último censo, hay que encontrar en cada caso un divisor $K$ (en este caso $K=46.000$ ) tal que:

$$
\sum_{i=1}^{4}\left[\frac{P_{i}}{K}\right]_{w}=2
$$

Los ocho escaños que faltan hasta los treinta, son los dos escaños iniciales que corresponden a cada una de las cuatro circunscripciones que componen la provincia de Santa Cruz de Tenerife.

De igual modo, para calcular los escaños que corresponden a cada una de las tres circunscripciones de Las Palmas mediante el método Webster y teniendo en cuenta el último censo, hay que encontrar en cada caso un divisor $K$ (en este caso $K=45.000$ ) tal que:

$$
\sum_{i=1}^{3}\left[\frac{P_{i}}{K}\right]_{w}=24
$$


Los seis escaños que faltan hasta los treinta, son los dos escaños iniciales que corresponden a cada una de las tres circunscripciones que componen la provincia de Las Palmas.

De este modo, el reparto de escaños en las islas (circunscripciones) quedaría como se muestra en la Tabla 6, donde se ha tenido en cuenta el Padrón de 2010 en las elecciones de 2011 para el reparto proporcional (aplicando el método Webster) de los escaños a las circunscripciones de cada una de las dos provincias.

TABLA 6. REPARTO DE ESCAÑOS A LAS CIRCUNSCRIPCIONES CON SAINTE-LAGUË

\begin{tabular}{|l|r|r|r|r|}
\hline \multirow{2}{*}{ Circunscripción } & \multirow{2}{*}{ Población } & \multirow{2}{*}{ Cuota } & \multicolumn{2}{c|}{ Escaños } \\
\cline { 4 - 5 } & & & Actuales & Propuesta \\
\hline Tenerife & 906854 & 25,68 & 15 & $\mathbf{2 2}(20+2)$ \\
\hline Gran Canaria & 845676 & 23,95 & 15 & $\mathbf{2 1}(19+2)$ \\
\hline Lanzarote & 141437 & 4,01 & 8 & $\mathbf{5 ( 3 + 2 )}$ \\
\hline Fuerteventura & 103492 & 2,93 & 7 & $\mathbf{4}(2+2)$ \\
\hline La Palma & 87324 & 2,47 & 8 & $\mathbf{4}(2+2)$ \\
\hline La Gomera & 22776 & 0,65 & 4 & $\mathbf{2}(0+2)$ \\
\hline El Hierro & 10960 & 0,31 & 3 & $\mathbf{2 ( 0 + 2 )}$ \\
\hline Total & 2118519 & 60 & 60 & 60 \\
\hline
\end{tabular}

Fuente: Elaboración propia a partir de Padrón de 2010 (www.ine.es) y página oficial del Parlamento de Canarias (www.parcan.es)

Con la biproporcionalidad se asignan los escaños a los partidos en función de sus votos totales. Por tanto, el tamaño de circunscripción no influye en la representación de los partidos. Para aplicar la biproporcionalidad vamos a tener en cuenta las siete circunscripciones actuales. Los tamaños de las siete circunscripciones serían los que aparecen en la Tabla 6. Para el cálculo del reparto de escaños a los partidos tendremos en cuenta sus votos totales con una barrera del $3 \%$ de los votos a candidaturas y usaremos el método d’Hondt (Tabla 7).

TABLA 7. REPARTO DE ESCAÑOS A LOS PARTIDOS CON BARRERA DEL 3\%

\begin{tabular}{|c|c|c|c|c|}
\hline \multirow{2}{*}{ Partido } & \multirow{2}{*}{ Votos } & \multirow{2}{*}{ Cuota } & \multicolumn{2}{|c|}{ Escaños } \\
\hline & & & Actuales & Propuesta \\
\hline$P P$ & 289381 & 19,71 & 21 & 22 \\
\hline$C C-P N C-C C N$ & 223785 & 15,24 & 20 & 17 \\
\hline PSOE & 190028 & 12,94 & 15 & 15 \\
\hline $\mathrm{NC}$ & 82148 & 5,60 & 3 & 6 \\
\hline ACSP & 19020 & 1,30 & & \\
\hline Verdes & 18831 & 1,28 & & \\
\hline UPD & 9069 & 0,62 & & \\
\hline $\mathrm{AHI}-\mathrm{CC}$ & 2163 & 0,15 & 1 & \\
\hline Otros & 46517 & 3,17 & & \\
\hline Total & 880942 & 60 & 60 & 60 \\
\hline
\end{tabular}

Fuente: Elaboración propia a partir de Padrón de 2010 (www.ine.es) y página oficial del Parlamento de Canarias (www.parcan.es)

En la Tabal 8 se recoge la distribución de votos entre partidos y circunscripciones, a partir de la cual se desarrolla el método de reparto biproporcional de la Tabla 9 en el cual aparecen los escaños de cada partido en cada circunscripción aplicando el método de Webster. En la Tabla 9 tenemos los partidos en la primera fila y las circunscripciones en la primera columna, dentro de la tabla aparecen los escaños que recibe cada partido en cada circunscripción, y en las marginales tenemos el total de escaños de cada partido (última fila) y el total de escaños de cada circunscripción (última columna). Estos valores constituyen una doble restricción por filas y por columnas, los cuales son las marginales de nuestro problema de reparto. Para conseguir resolver este problema de reparto existe la mencionada técnica de la biproporcionalidad, la cual aplicamos en este caso. 
TABLA 8. REPARTO DE VOTOS ENTRE PARTIDOS Y CIRCUNSCRIPCIONES

\begin{tabular}{|l|r|r|r|r|r|}
\hline Circunscripción & \multicolumn{1}{|c|}{ PP } & CC-PNC-CCN & \multicolumn{1}{c|}{ PSOE } & \multicolumn{1}{c|}{ NC } & \multicolumn{1}{c|}{ Total } \\
\hline Tenerife & 99673 & 142401 & 78645 & 18205 & 338924 \\
\hline Gran Canaria & 156200 & 34931 & 79644 & 53893 & 324668 \\
\hline Lanzarote & 9582 & 14901 & 7386 & 5840 & 37709 \\
\hline Fuerteventura & 6885 & 11380 & 5842 & 2915 & 27022 \\
\hline La Palma & 12577 & 17700 & 11133 & 795 & 42205 \\
\hline La Gomera & 2931 & 2472 & 5324 & 500 & 11227 \\
\hline Hierro & 1533 & & 2054 & & 3587 \\
\hline Total & 289381 & 223785 & 190028 & 82148 & 785342 \\
\hline
\end{tabular}

Fuente: Elaboración propia a partir de la página oficial del Parlamento de Canarias (www.parcan.es)

TABLA 9. REPARTO BIPROPORCIONAL DE ESCAÑOS ENTRE PARTIDOS Y CIRCUNSCRIPCIONES

\begin{tabular}{|c|c|c|c|c|c|}
\hline Circunscripción & PP & CC-PNC-CCN & PSOE & NC & Total \\
\hline Tenerife & 7 & 9 & 5 & 1 & 22 \\
\hline Gran Canaria & 10 & 2 & 5 & 4 & 21 \\
\hline Lanzarote & 1 & 2 & 1 & 1 & 5 \\
\hline Fuerteventura & 1 & 2 & 1 & & 4 \\
\hline La Palma & 1 & 2 & 1 & & 4 \\
\hline La Gomera & 1 & & 1 & & 2 \\
\hline Hierro & 1 & & 1 & & 2 \\
\hline Total & 22 & 17 & 15 & 6 & 60 \\
\hline
\end{tabular}

Fuente: Elaboración propia a partir de la página oficial del Parlamento de Canarias (www.parcan.es)

Con el método de la biproporcionalidad se trata de conseguir una doble proporcionalidad: para las circunscripciones cuyo tamaño se ha obtenido en función de su población actualizada, y para los partidos cuyo número total de escaños se ha obtenido en función de sus votos totales (Ramírez González, Pukelsheim, Palomares Bautista y Martínez Aroza, 2008).

En un reparto proporcional se tienen, por ejemplo, los votos de varios partidos y hay que multiplicarlos por un factor para aplicarle después un redondeo a cantidades enteras, que sumen el número de escaños a repartir. En un reparto biproporcional se tiene una tabla de votos y hay que multiplicar los votos de cada fila de la tabla y los votos de cada columna de la tabla por factores de tal manera que los redondeos a cantidades enteras sumen por filas y por columnas lo que se indica en las marginales que aparecen junto a los nombres de los partidos y de las circunscripciones. Este problema tiene solución, pero su cálculo es complejo y lo más fácil es recurrir a un programa informático como el BAZI (Maier y Pukelsheim, 2007).

\section{5-. CONCLUSIONES}

Un sistema electoral idóneo ha de compaginar representatividad y gobernabilidad o capacidad de generar gobiernos estables (Bilbao Arrese, 1994; Urdánoz Ganuza, 2004). La distribución de escaños a los partidos para las elecciones canarias en las siete circunscripciones (islas) mediante repartos independientes ha conducido de manera sistemática a que aparezcan discordancias entre votos totales y escaños totales. Estas discordancias se ven reforzadas en ocasiones por la actual distribución de los escaños entre las circunscripciones electorales, la cual ha sido invariable a lo largo de las tres décadas de democracia, sin tener en cuenta sus poblaciones actualizadas. Esa distribución prima a las islas pequeñas (Lanzarote, Fuerteventura, La Palma, La Gomera, El Hierro) frente a las dos más grandes (Tenerife y Gran Canaria). Como consecuencia beneficia a los partidos o coaliciones nacionalistas frente a otros partidos de ámbito nacional como PP y PSOE que tienen mayor implantación en Tenerife y Gran Canaria.

Nuestra propuesta de sistema electoral usa como circunscripción electoral las actuales siete islas y presenta dos ventajas frente al sistema usado en la actualidad. En primer lugar, con nuestra propuesta de una nueva y única barrera electoral del $3 \%$ de los votos a candidaturas a nivel regional, se fortalece la gobernabilidad y se evitan 
las discordancias entre votos y escaños causados por la doble barrera actual y por los repartos independientes. Además el partido vencedor obtiene unos resultados similares a los obtenidos con el sistema electoral actual (Tabla 7). En segundo lugar, se produce una gran equidad en el valor del voto de los electores, es decir, el voto recibido por un partido tiene exactamente el mismo valor sea cual sea la circunscripción en la que se emita. Además un partido con más votos que otro nunca recibe menos escaños. En definitiva, se consigue alta representatividad y se favorece la gobernabilidad. Todo ello es posible porque el sistema electoral propuesto contempla en la última etapa un reparto biproporcional. La técnica de la briproporcionalidad ya fue usada por primera vez en 2006 en el cantón suizo de Zurich y posteriormente en 2008 en los cantones de Argovia y de Escafusa.

\section{AGRADECIMIENTOS}

Los autores desean agradecer a la Junta de Andalucía las ayudas recibidas a través del Proyecto de Excelencia SEJ-8044 y a través de las ayudas al grupo FQM-0191.

\section{BIBLIOGRAFÍA}

BALINSKI, Michel y DEMANGE, Gabrielle (1989): “An axiomatic approach to proportionality between matrices", Mathematics of Operations Research, n¹4, págs. 700-719.

BILBAO ARRESE, Mario (1994): “Ley electoral y sistema de partidos en España”, Revista de Estudios Políticos, n 85 , págs. 313-321.

DELGADO RAMOS, David (2011): “Elecciones al Parlament 2010: fin de ciclo en Cataluña”, Revista de Derecho Político (UNED), $\mathrm{n}^{\circ} 80$, págs. 201-234.

ESTATUTO DE AUTONOMÍA DE CANARIAS. Ley Orgánica 10/1982, de 10 de agosto, reformada por Ley Orgánica 4/1996, de 30 de diciembre.

FALCÓ GIMENO, Alberto y VERGE MESTRE, Tania (2013): “Coalition Trading in Spain: Explaining State-wide Parties' Government Formation Strategies at the Regional Level”, Regional and Federal Studies, vol. 4, n²3, págs. 387-405.

GAMBINO, Silvio (2009): "Relaciones entre sistema electoral, formato de partidos y forma de gobierno en la experiencia parlamentaria española”, Revista de Estudios Políticos (Nueva Época), n 146, págs. 11-47.

GÓMEZ FORTES, Braulio y CABEZA PÉREZ, Laura (2013): “Basque Regional Elections 2012: The Return of Nationalism under the Influence of the Economic Crisis”, Regional and Federal Studies, vol 4, n² 23, págs. $495-505$.

LAGO PEÑAS, Ignacio y LAGO PEÑAS, Santiago (2000): “El sistema electoral español: una cuantificación de sus efectos «mecánico» y «psicológico»”, Revista de Estudios Políticos (Nueva Época), n 107, págs. 225 -250.

LAGO PEÑAS, Ignacio y MONTERO GIBERT, José Ramón (2004): “Más votos y menos escaños: El impacto del sistema electoral en las elecciones autonómicas catalanas de 2003”, Revista de Estudios Políticos (Nueva Época), $\mathrm{n}^{\circ} 105$, págs. 11-42.

LEY ORGÁNICA 10/1982, de 10 de agosto, de Estatuto de Autonomía de Canarias.

LEY 7/2003, de 20 de marzo, de elecciones al Parlamento de Canarias (B.O.C. 57, de 24.3.2003).

LIBBRECHT, Liselotte; MADDENS, Bart y SWENDEN, Wilfried (2011): "Party competition in regional elections: The strategies of state-wide parties in Spain and the United Kingdom”, Party Politics, vol 4, núm. 19, págs. 624-640.

MAIER, Sebastian y PUKELSHEIM, Friedrich (2007): Bazi: A free computer program for proportional representation apportionment, Augsburg: Institut für Mathematik.

MONTERO GIBERT, José Ramón (1997): “El debate sobre el sistema electoral: Rendimientos, criterios y propuestas de reforma”, Revista de Estudios Políticos (Nueva Época), n 95, págs. 9-46.

MONTERO GIBERT, José Ramón y FONT FABREGAS, Joan (1991): “El voto dual en Cataluña: Lealtad y transferencia de votos en las elecciones autonómicas”, Revista de Estudios Políticos (Nueva Época), n²73, págs. $7-34$.

MONTERO GIBERT, José Ramón y RIERA SAGRERA, Pedro (2008): “Informe sobre la Reforma del Sistema Electoral”, presentado a la Comisión de Estudios del Consejo de Estado en Diciembre de 2008.

MONTERO GIBERT, José Ramón y RIERA SAGRERA, Pedro (2009): “El sistema electoral español: Cuestiones de desproporcionalidad y de reforma", Anuario de la Facultad de Derecho de la Universidad Autónoma de Madrid, $\mathrm{n}^{\circ} 13$, págs. $225-270$. 
NOHLEN, Dieter (1983): "Reforma del sistema electoral español: Conveniencias, fórmulas y efectos políticos”, Revista de Estudios Políticos (Nueva Época), n³ 34, págs. 61-68.

OÑATE RUBALCABA, Pablo y OCAÑA LARA, Francisco A. (2000): “Elecciones de 2000 y sistemas de partidos en España: ¿Cuánto cambio electoral?”, Revista de Estudios Políticos (Nueva Época), n 110, págs. $297-336$.

PADRÓ SOLANET, Albert y COLOMER CALSINA, Josep Maria (1992): “Espacio político-ideológico y temas de campaña”, Revista de Estudios Políticos (Nueva Época), nº 78, págs. 131-159.

PALLARÉS PORTA, Francesc (1981): “La distorsión de la proporcionalidad en el sistema electoral español. Análisis comparado e hipótesis alternativas”, Revista de Estudios Políticos (Nueva Época), n²3, págs. $233-267$.

PÉREZ ALBERDI, María Reyes (2001): “Efectos de las barreras electorales. Estudio del sistema electoral canario a raíz de la STC 225/1998”, Revista de Derecho Político, n 52, págs. 357-401.

PROPOSICIÓN DE LEY PARA LA DEMOCRATIZACIÓN DEL SISTEMA ELECTORAL AL PARLAMENTO DE CANARIAS, 22 de diciembre de 2010. Boletín Oficial del Parlamento de Canarias, VII Legislatura núm. 401.

PROPOSICIÓN DE LEY. PROPUESTA DE REFORMA DEL ESTATUTO DE AUTONOMÍA DE CANARIAS, Presentada por el Parlamento de Canarias. 22 de Septiembre 2006, Boletín Oficial de las Cortes Generales, $n^{\circ}$ 621-1, serie B.

RAMÍREZ GONZÁLEZ, Victoriano (2010): “Proposta di riforma del sistema elettorale per la Camera in Italia”, POLENA (Political and Electoral Navigations), n², págs. 85-94.

RAMÍREZ GONZÁLEZ, Victoriano y MÁRQUEZ GARCÍA, Ana (2010): “Un sistema electoral ecuánime para el Congreso de los Diputados”, Revista Española de Ciencia Política, n² 24, págs. 139-160.

RAMÍREZ GONZÁLEZ, Victoriano; PUKELSHEIM, Friedrich; PALOMARES BAUTISTA, Antonio y MARTÍNEZ AROZA, José Antonio (2008): "A bi-proportional method applied to the Spanish congress", Mathematical and Computer Modelling, n 48, págs. 1461-1467.

RAMÍREZ GONZÁLEZ, Victoriano et al. (2013): Sistema electoral para el Congreso de los Diputados: Propuesta para un parlamento más ecuánime, representativo y gobernable, Granada: Editorial de la Universidad de Granada.

RIERA SAGRERA, Pedro (2013): “Los sistemas electorales y la cigüeña. Sobre el origen y la reforma de las reglas del juego democrático”, Revista Española de Investigaciones Sociológicas, n 142, págs. 141-150.

VALLÈS CASADEVALL, Josep Maria (1986): "Sistema electoral y democracia representativa: Nota sobre la Ley Orgánica del Régimen General de 1985 y su función política”, Revista de Estudios Políticos (Nueva Época), nº 53 , págs. 7-28.

SCHAKEL, Arjan H. (2011): "Congruence between regional and national elections", Comparative Political Studies, vol 5, n 46, págs. 631-662.

SENTENCIA 225/1998, de 25 de noviembre de 1998, Recurso de inconstitucionalidad 1.324/1997, Boletín Oficial del Estados (BOE), nº 312, págs. 18-27.

\section{WEBGRAFÍA}

Canarias Ahora. El periódico digital de Canarias. 2013. "Un sistema injusto y desproporcional." Consulta 26 de Agosto de 2013 (www.canariasahora.es/articulo/canarias/nc-pide-altura-democratica-abordar-reforma-leyelectoral/20130826164659412363.html)

Centro Canario Nacionalista (CCN) . 2013. "El CCN felicita a Nueva Canarias por defender la reforma del sistema electoral”. Consulta 1 de Octubre de 2013 (www.centrocanario.org/actualidad/canarias/71-poltica/665-elccn-felicita-a-nueva-canarias-por-defender-la-reforma-del-sistema-electoral)

Demócratas para el Cambio: Asociación Cívica para la Reforma Electoral Canaria. 2007. “El sistema electoral canario y su reforma." (www.democratasparaelcambio.com)

Instituto Nacional de Estadística (INE): www.ine.es

Nueva Tribuna. 2013. “Una reforma electoral para Canarias.” Regidor Arenales, Mario Marcelo. Consulta 16 Octubre de 2013 (www.nuevatribuna.es/opinion/mario-regidor/reforma-electoral-canarias/20131015183804097398. html) 
Página Oficial del Parlamento de Canarias: www.parcan.es

Partido Socialista Obrero Español (PSOE). 2009. "Reforma del Sistema Electoral Canario." Consulta 8 de Octubre de 2009 (www.psoe.es//ambito/canarias/docs/index.do?action=View\&id=401527)

Wikimedia Commons: (http://commons.wikimedia.org/wiki/File:Mapa_de_Canarias.png) 
